\title{
THE DETERMINATION OF THE SENSITIVITY OF MYCO. TUBERCULOSIS TO STREPTOMYCIN
}

\author{
BY \\ R. W. FAIRBROTHER AND J. E. SOUTHALL \\ From the Department of Clinical Pathology, Manchester Royal Infirmary
}

(RECEIVED FOR PUBLICATION NOVEMBER 24, 1950)

Streptomycin is now widely used for the treatment of tuberculosis, and, as the development of resistance by tubercle bacilli is a common occurrence in certain forms of the disease, bacteriological control is important. There is consequently a definite need for a reliable and simple routine method of testing the sensitivity to streptomycin of the tubercle bacillus as the demand for the information is increasing steadily.

The method in general use requires the serial dilution of streptomycin in the fluid Tween-albumin medium, described by Dubos and Davis (1946) and in the M.R.C. report (Lancet, 1948). This has given consistent results, but the technique is complicated and time-consuming and there is considerable delay (six to eight weeks) in obtaining the results. Other fluid media, such as the synthetic medium of Proskauer and Beck (surface and deep), have not generally proved as satisfactory as the Dubos method although giving comparable results (Wolinsky and Steenken, 1947).

Various forms of solid media, in particular Herrold's medium, have been introduced, and it is claimed that they provide a rough estimate of the sensitive and resistant organisms in the inoculum; this information is not given by fluid media (Pyle, 1947 ; Karlson and Needham, 1948). Herrold's egg-yolk medium does not appear to have been widely used in this country. Holt and Cruickshank (1949) tried Lowenstein and Jensen's medium, containing only approximate amounts of streptomycin, and reported satisfactory results from the examination of sputum. Youmans, Ibrahim, Sweany, and Sweany (1950) carried out direct sensitivity tests on more than 2,000 sputum examples and considered that more accurate results were obtained with Herrold's medium than with inspissated media, in which the final concentration of streptomycin was only approximate owing to the heating of the medium after the addition of the antibiotic. Williston and Youmans (1949) tested a wide range of media and demonstrated that the type of medium had a definite influence on the sensitivity results ; they considered that fluid media, in particular the original Dubos medium, had many disadvantages and recommended Herrold's medium for general use. The slide method of Pryce (1941) has been tried, but it seems to have distinct limitations as a routine test; the inoculum must contain large numbers of tubercle 
bacilli and so direct tests with pathological material may give erratic results (Cummings and Drummond, 1949).

During the past three years sensitivity tests have been carried out on strains of Myco. tuberculosis isolated from tuberculous material, mainly cerebrospinal fluid, by means of the standard Dubos technique; trials with various solid media have also been made. The results are now reported.

\section{Tween-albumin (Dubos) Medium}

Technique.-The method recommended in the M.R.C. report (1948) has been closely followed ; glucose and vegex were not added to the medium in which the final concentration of the bovine albumin was $0.3 \%$. Primary isolation of the organisms was carried out either on the Lowenstein-Jensen medium or at times by guinea-pig inoculation, from which further cultures, and if necessary subcultures, were prepared in the Dubos medium in order to obtain a diffuse growth; the concentration of organisms was adjusted to an opacity roughly equivalent to tube No. 2 of the Brown opacity series and a 1/10 dilution was prepared for the assay. The standard strain, H37Rv, was used as the control in each series of tests. Readings were made after incubation at $37^{\circ} \mathrm{C}$. for 10 and 14 days.

\section{Results}

Eighty-one strains of Myco. tuberculosis have been examined ; these were isolated from 56 strains of tuberculosis - 49 meningitis, five adenitis, and two pulmonary. Sixtysix strains were obtained from the cerebrospinal fluid and include 17 strains isolated at varying stages of streptomycin therapy, in some instances nine to 12 months after the beginning of the treatment ; six strains were obtained at necropsy from lesions at the base of the brain (Table I).

\section{TABLE I}

Sensitivity of Myco. tuberculosis to StREPTOMycin (81 Strains)

\begin{tabular}{|c|c|c|c|c|c|c|}
\hline Inhibited by $\frac{1}{2} \mu \mathrm{g}$. or less & & & $\cdots$ & $\cdots$ & . & . \\
\hline "1 $1 \mu \mathrm{g}$. & $\cdots$ & $\cdots$ & ・• & - & $\cdots$ & .. \\
\hline ,2 $2 \mu \mathrm{g}$. & - & . & . & .. & . & - \\
\hline ibited by $600 \mu \mathrm{g}$. & $\ddot{0}$ & . & - & - & . & . \\
\hline
\end{tabular}

The results, in general, agree with those obtained by other workers (M.R.C. Report, 1948 ; Mitchison, 1949). Strains isolated before streptomycin therapy proved very sensitive, while the meningitis strains did not tend to develop resistance during treatment.

The resistant strain was isolated from a kidney removed at nephrectomy of a case of meningitis developing a renal lesion during the course of treatment; at the same time Myco. tuberculosis was also isolated from the cerebrospinal fluid, but this strain proved to be sensitive. It is therefore important to note that strains isolated from one particular focus of a widespread infection do not necessarily indicate the sensitivity of strains in the other lesions.

This method has given consistent results, but it has the great disadvantage of taking some six to eight weeks before the final result is available. It is usually necessary to institute treatment before the sensitivity results are available. 


\section{Solid Media}

Two types of solid media have been tried: (1) fluid Dubos solidified by the addition of agar, and (2) Herrold's egg-yolk medium. These have been used in either petri dishes or screw-cap bottles as a slope.

Technique with Dubos Medium.-Agar (Davis) $1 \%$ was added to the fluid medium and $2.5-\mathrm{ml}$. amounts were distributed in bijou bottles before the addition of the bovine albumin. The medium was sterilized in the autoclave for 10 minutes at $10 \mathrm{lb}$. pressure. The melted medium was placed in the $56^{\circ} \mathrm{C}$. bath and the sterile albumin solution added to each bottle together with the appropriate amount of the standard streptomycin solution, the final strengths of which were $0.25,0.5,1,2,5$, and $10 \mu \mathrm{g}$. per ml. Petri dishes containing $15 \mathrm{ml}$. of the medium were also prepared using a similar technique.

Technique with Herrold's Medium.-The original formula was closely followed (Herrold, 1931a and b). This is :

$$
\begin{aligned}
& \begin{array}{llllllllll}
\text { Lab. Lemco } & \ldots & \ldots & \ldots & \ldots & \ldots & \ldots & \ldots & \ldots & 3 \text { g. }
\end{array} \\
& \begin{array}{lllllllll}
\text { Peptone (Evans) } & \ldots & \ldots & \ldots & \ldots & \ldots & \ldots & \ldots & 10 \text { g. }
\end{array}
\end{aligned}
$$

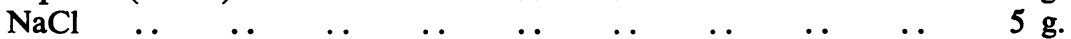

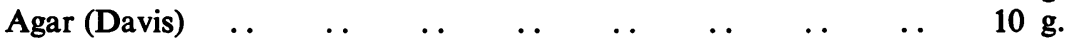

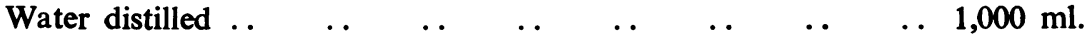

The $p \mathrm{H}$ is adjusted to 7.5 , and $150-\mathrm{ml}$. amounts are distributed in $250-\mathrm{ml}$. flasks and sterilized by autoclaving for 15 minutes at $10 \mathrm{lb}$. pressure. Glycerin was not added.

The yolk of one fresh egg was added to each $150-\mathrm{ml}$. batch of medium at $56^{\circ} \mathrm{C}$., making a final concentration of approximately $15 \%$ of egg yolk ; the appropriate amounts of the standard streptomycin solution were carefully added at this stage; on cooling to $45^{\circ} \mathrm{C}$. the medium was distributed in petri dishes or, as slopes, in bijou bottles $(2.5 \mathrm{ml}$.).

In the meantime eggs, which should be fresh, were washed well first with soap and warm water and then with hot tap water, after which they were placed in methylated spirits for 15 minutes; they were then dried individually with a sterile cloth and placed in a sterile petri dish. A small opening was made at one end of the egg with sterile forceps and the white of the egg removed. The hole was next enlarged and the yolk poured into a sterile beaker, in which it was broken up with a sterile glass rod and then added to the agar base at $56^{\circ} \mathrm{C}$.

The media were seeded and incubated at $37^{\circ} \mathrm{C}$. for at least 14 days. This presented no difficulty in the case of the screw-cap bottles, but considerable care was necessary with the petri dishes in order to obtain satisfactory results. A convenient and simple procedure was to wrap each plate in paper and place in a covered glass jar.

\section{Results}

(A) Solid Dubos. - This proved a good medium for Myco. tuberculosis; growth on the streptomycin-free medium was apparent in some six days and became profuse in 10 to 14 days.

Parallel tests were made with the solid and fluid Dubos media using pure cultures of Myco. tuberculosis. The results given by the two types of media were in close agreement, i.e., with sensitive strains growth was inhibited by the presence of $1 \mu \mathrm{g}$. per ml. of streptomycin.

Attempts to obtain quick results were made by the direct seeding of the media with tuberculous material collected from patients. It is, however, essential that large numbers of tubercle bacilli should be present in the inoculum, and it is therefore not 
surprising that cerebrospinal fluid proved unsatisfactory owing to the scanty numbers of bacilli present ; good results were, however, obtained with caseous material from human, as well as guinea-pig, sources by this technique.

In the case of cerebrospinal fluid, it was necessary first to isolate the tubercle bacilli on Lowenstein-Jensen medium and then to subculture in the Dubos fluid medium, which was suitable for inoculation when a general turbidity was apparent. The time saved in obtaining the final report of the sensitivity of a strain was therefore of little practical value.

(B) Herrold's Medium.-When freshly prepared, this proved an excellent medium for the tubercle bacillus; obvious growth developed in three to five days and was luxuriant in seven to ten days.

Sensitivity tests were made mainly on pure cultures of tubercle bacilli, usually in the fluid Dubos medium, using (1) media in which varying amounts of streptomycin were incorporated, or (2) the filter-paper technique. Direct assays were made on a few samples of sputum from tuberculous patients.

Streptomycin was incorporated in the medium to give a final concentration of $0.5,1,2,5$, and $10 \mu \mathrm{g}$. per ml. Comparative tests were made with the fluid and solid Dubos media. The results showed .general agreement. It was, however, interesting to note that inhibition of growth was obtained by the presence of $1 \mu \mathrm{g} . / \mathrm{ml}$. in the solid Dubos medium, but in the case of the Herrold medium the addition of at least $2 \mu \mathrm{g} . / \mathrm{ml}$. was frequently required to produce this result. The influence of the medium on the sensitivity of tubercle bacilli has already been noted (Williston and Youmans, 1949). It is therefore important that, when the results of sensitivity tests are given in terms of units or $\mu \mathrm{g}$., the medium used in the assay should be stated.

The preparation of the Herrold medium is relatively simple, and, when petri dishes are used, resistant colonies can be readily selected and, if necessary, several strains can be tested on one plate. It is also possible to obtain a quick result by direct plating when the inoculum contains many tubercle bacilli as in the case of many samples of sputum. These are distinct advantages over the fluid media.

The use of filter-paper strips or disks has proved useful as a crude and simple method of testing the sensitivity of organisms to the antibiotics, and it is widely used in routine practice. An attempt was therefore made to adapt this technique to the study of the tubercle bacillus. Disks were impregnated with varying concentrations of streptomycin-10,20,50,100,250,500, and $1,000 \mu \mathrm{g} . / \mathrm{ml}$.- and then placed carefully on the Herrold media which had been heavily seeded with sensitive strains of $M y c o$. tuberculosis. Incubation at $37^{\circ} \mathrm{C}$. was carried out for two to three weeks. No effect was observed with strengths up to $100 \mu \mathrm{g} . / \mathrm{ml}$. , but definite zones of inhibitions were produced by concentrations of $250 \mu \mathrm{g} . / \mathrm{ml}$. and greater.

A concentration of $500 \mu \mathrm{g} . / \mathrm{ml}$. was consequently adopted for the routine tests, in which strips of filter paper were mainly used. This proved a convenient method of testing several strains at the same time and obtaining a crude index of sensitivity of the organisms to streptomycin (Figs. 1 and 2).

Minor variations in sensitivity cannot, however, be detected by this simple technique. Cultures in a fluid medium are advisable, and there is therefore little, if any, saving of time, but, as several strains can be tested on one plate, it provides a relatively simple and economic technique. 
The relative simplicity of the solid media, particularly the strip technique, should prove useful as a screening test in routine laboratories. Strains giving irregular results by these tests could then be referred for a more complete assay to central laboratories, where the elaborate techniques are available.

\section{Summary}

The fluid Tween-albumin medium has given consistent results with strains of Myco. tuberculosis isolated mainly from cases of meningitis.

Solid media, in the form of Dubos or Herrold, have also given reliable results, but the time saved by their use in cases of tuberculous meningitis is of little practical value. They are particularly useful for the direct examination of material likely to contain large numbers of tubercle bacilli.

The filter-paper technique, using a streptomycin concentration of $500 \mu \mathrm{g}$. per ml., provides a simple, crude method of assaying sensitivity.

It is a pleasure to thank the Medical Research Council and Dr. J. Ungar for the supply of standard streptomycin and the Department of Clinical Photography for the photographs.

\section{REFERENCES}

Cummings, M., and Drummond, M. (1949). Amer. Rev. Tuberc., 59, 599.

Dubos, R. J., and Davis, B. D. (1946). J. exp. Med., 83, 409.

Herrold, R. D. (1931a). J. infect. Dis., 48, 236.

- (1931b). Ibid., 49, 420.

Holt, H. D., and Cruickshank, R. (1949). Mon. Bull. Min. Hlth, 8, 103.

Karlson, A. G., and Needham, G. M. (1948). Proc. Mayo Clin., 23, 401.

Medical Research Council (1948). Lancet, $2,862$.

Mitchison, D. A. (1949). Ibid., 2, 694.

Pryce, D. M. (1941). J. Path. Bact., 53, 327.

Pyle, M. M. (1947). Proc. Mayo Clin., 22, 465.

Williston, E. H., and Youmans, G. P. (1949). Amer. Rev. Tuberc., 59, 336.

Wolinsky, E., and Steenken, W., jun. (1947). Ibid., 55, 281.

Youmans .G. P., Ibrahim, A., Sweany, J., and Sweany, H. C. (1950). Ibid., 61, 569. 